\title{
Jagüeyes y su papel potencial en la conservación de tortugas continentales en el golfo de Morrosquillo, Sucre, Caribe colombiano
}

\author{
Cattle ponds and their potential role in conservation of freshwater turtles in the Gulf of \\ Morrosquillo, Sucre, Colombia
}

\author{
Jaime De La Ossa-V., Merly Ardila-Marulanda, Alejandro De La Ossa-Lacayo
}

\begin{abstract}
Resumen
El presente trabajo analiza la ocupación territorial de jagüeyes (pequeñas lagunas de origen antrópico) por quelonios, discute y plantea la relación que este hábitat tiene con la conservación local en una zona antropizada del golfo de Morrosquillo, Sucre, Colombia. Se seleccionaron 65 jagüeyes con área mayor a $1.000 \mathrm{~m}^{2}$, y se trabajó con una muestra representativa de 12 jagüeyes escogidos al azar y sin comunicación entre ellos. La toma de información poblacional se realizó mediante muestreos llevados a cabo entre enero y marzo de 2014, entre las 08:00 y las 12:00 horas, utilizando un trasmallo $100 \mathrm{~m}$ de largo por $6 \mathrm{~m}$ de ancho y ojo de $5 \mathrm{~cm}$ y en cada sesión se hicieron dos barridos, manteniendo un cubrimiento del $90 \%$ en los arrastres del área de cada jagüey. Se capturó una población total de 383 quelonios, 241 Trachemys callirostris; 66 Kinosternon scorpiodes y 76 Mesoclemmys dahli. El cálculo de población estimada para cada especie mediante el Método de Lincoln Petersen dio como resultado un tamaño estimado de 259 T. callirostris, $82 \mathrm{~K}$. scorpiodes y $62 \mathrm{M}$. dahli.
\end{abstract}

Palabras clave. Golfo de Morrosquillo. Humedales. Lagunas artificiales ganaderas. Ocupación. Quelonios.

\begin{abstract}
This study analyzed the territorial occupation of cattle ponds by turtles and established the relationship that this habitat has with local conservation in a human impacted zone in the Gulf of Morrosquillo, Sucre, Colombia. Sixty-five cattle ponds were selected, with an area greater than $1,000 \mathrm{~m}^{2}$; and a representative sample of 12 randomly selected waterholes that were not connected was used. The population data were collected with samples taken between January and March of 2014, between 08:00 and 12:00 p.m., using a trammel net that was $100 \mathrm{~m}$ long and $6 \mathrm{~m}$ wide with $5 \mathrm{~cm}$ mesh. Two sweeps were carried out for each session, covering 90 $\%$ of the area of each pond. A population of 383 individuals was obtained: 241 Trachemys callirostris, 66 Kinosternon scorpioides, and 76 Mesoclemmys dahli. The estimated population calculated for each species was obtained with the Lincoln-Petersen method, obtaining estimated populations of 259 for T. callirostris, 82 for K. scorpiodes and 62 for M. dahli.
\end{abstract}

Key words. Artificial cattle ponds. Chelonian. Morrosquillo Gulf. Occupation. Wetlands. 


\section{Introducción}

Los jagüeyes, unidades construidas como abrevaderos de ganado, funcionan como pequeños humedales con un elevado grado de naturalidad y poseen una riqueza de especies que los hacen especialmente valiosos para la conservación (León et al. 2010, Casas et al. 2012). Permiten evidenciar que existe una relación positiva entre el número de jagüeyes y la biodiversidad local de un área dada, en especial en zonas de bosque seco y en el cinturón árido pericaribeño (Botero et al. 2009). Las charcas ganaderas o jagüeyes, son ecosistemas antrópicos, lénticos y someros, se encuentran principalmente en zonas de clima árido y semiárido. Su llenado se produce mayormente a partir del agua de lluvia por lo que su periodo de inundación o hidroperiodo puede resultar muy irregular intra e interanualmente (Sancho y Lacomba 2010). En la mayor parte de los casos son sistemas de pequeña entidad, de carácter artificial o seminatural, utilizados $\mathrm{y}$ mantenidos tradicionalmente como fuentes de agua en zonas secas del Caribe colombiano. La alimentación de los mismos se genera mediante la creación de una zona o cuenca de captación del agua de lluvia, destinada a la generación de escorrentías, inserta en una matriz paisajística forestal o agrícola (Verdiell-Cubedo 2012).

En los jagüeyes del Caribe colombiano hay una ocupación territorial faunística constante, que incluye a los quelonios. Los mecanismos seguidos para la colonización de estos hábitats artificiales son distinguibles tanto en época de lluvias cuando los cauces temporales generalmente conectan los jagüeyes entre sí, y en época seca cuando por la ausencia de cuerpos de aguas disponibles, debido a la sequía, se da permanencia in situ o migraciones hacia los que aún conservan parte de su espejo de agua, convirtiéndose en refugios de gran valor ecológico (De La Ossa y De La Ossa-Lacayo 2013). Se dan entonces procesos de migración relacionados con los ciclos de lluvias, caracterizados por concentración durante la sequía y por migración y ocupación en época de precipitaciones (De La Ossa-Lacayo 2014), que ponen de manifiesto la capacidad de sobrevivencia exitosa de muchas especies silvestres regionales (Botero et al. 2009).
Para el golfo de Morrosquillo, Sucre, Colombia se tiene evidencia de la presencia de tres especies de quelonios continentales: Trachemys callirostris (hicotea) (Medem 1962); Kinosternon scorpiodes (tapaculo) (Rueda-Almonacid et al. 2007) y Mesoclemmys dahli (carranchina), endémica de la costa Caribe de Colombia, reconocida para los departamentos de Sucre, Córdoba, Bolívar, Atlántico, Cesar y Magdalena (Medem 1966, Rueda-Almonacid et al. 2007; Páez et al. 2012).

Por otro lado, el estatus nacional de cada una de estas especies, según Castaño-Mora 2002, CITES 2016 (https://cites.org/sites/default/files/eng/app/2016/SAppendices-2016-03-10.pdf), es: T. callirostris: $\mathrm{Vu}$ (Criterios) y CITES no listada. K. scorpiodes LC; CITES no listada y $M$. dahli: EN (actualizar criterios), CITES: no listada. De acuerdo con Morales-Betancourt et al (2015), T. callirostris y $K$. scorpiodes VU, mientras que $M$. dahli se le cataloga como EN.

El presente trabajo analiza la ocupación territorial de jagüeyes por las tres especies de quelonios continentales registrados para el área de estudio, discute y plantea la relación que este hábitat antrópico en relación a la conservación local en un escenario cuya matriz paisajística es netamente agropecuaria.

\section{Material y métodos}

Área de estudio. Se localiza entre los municipios de San Onofre y Santiago de Tolú, departamento de Sucre, Colombia $\left(9^{\circ} 41^{\prime} 40^{\prime \prime} \mathrm{N}-75^{\circ} 37^{\prime} 13^{\prime \prime} \mathrm{O}\right.$, $9^{\circ} 44^{\prime} 50^{\prime \prime} \mathrm{N}-73^{\circ} 31^{\prime} 13^{\prime \prime} \mathrm{O}, 9^{\circ} 35^{\prime} 30^{\prime \prime} \mathrm{N}-75^{\circ} 24^{\prime} 14^{\prime \prime}$ $\mathrm{O}, 9^{\circ} 28^{\prime} 53^{\prime \prime} \mathrm{N}-75^{\circ} 30^{\prime} 20^{\prime \prime} \mathrm{O}$ ) (Figura 1), con un área total de 17.088 ha. La zona pertenece al bioma tropical alternohígrico y hace parte del cinturón árido pericaribeño (Hernández-Camacho y Sánchez-Páez 1992), y se tipifica como bosque seco tropical (Bs-T) (Holdridge 1967). El Clima se clasifica típicamente Tropical, con precipitación anual entre 900 y 1.200 $\mathrm{mm}$, con temperatura media anual de $25^{\circ} \mathrm{C}$ y humedad relativa media del $80 \%$ (De La Ossa-Lacayo 2014). 


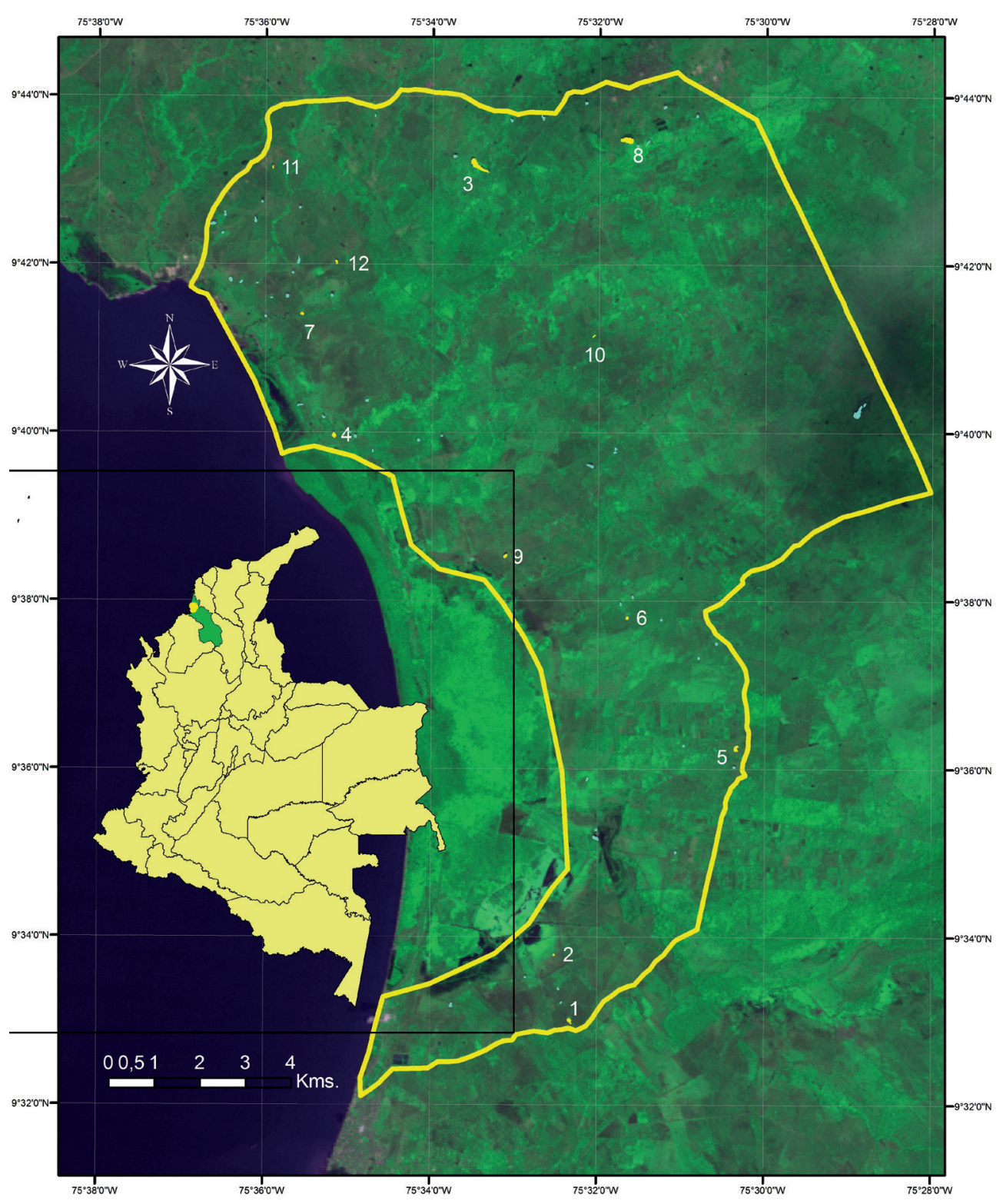

Figura 1. Mapa del área de estudio.

Muestreos: La ubicación inicial del área de estudio y todos los jagüeyes existentes, así como las medidas para calcular su área y su ubicación se hicieron mediante la aplicación del programa Google Earth (versión libre 7.1.2.2041), con imagen del 07-2013. Luego se llevaron a un formato Keyhole Markup Language ( $\mathrm{kml})$; todos los polígonos se trasladaron al formato shape (.shp) para ser utilizados en el software ArcMap de ArcGIS licenciado a la Universidad de
Sucre. Para el espacio mapa que señala los jagüeyes seleccionados para el estudio se trabajo con una imagen Landsat 5 TM (versión libre) del año 2011 con combinación de bandas 342. Se seleccionaron todos los polígonos con área mayor a $1.000 \mathrm{~m}^{2} \mathrm{de}$ espejo de agua, que no tuvieran comunicación entre ellos, dando como resultado un total de 65 jagüeyes, correspondiente a un área total de 24,07 ha (De La Ossa-Lacayo 2014) (Figura 2). 

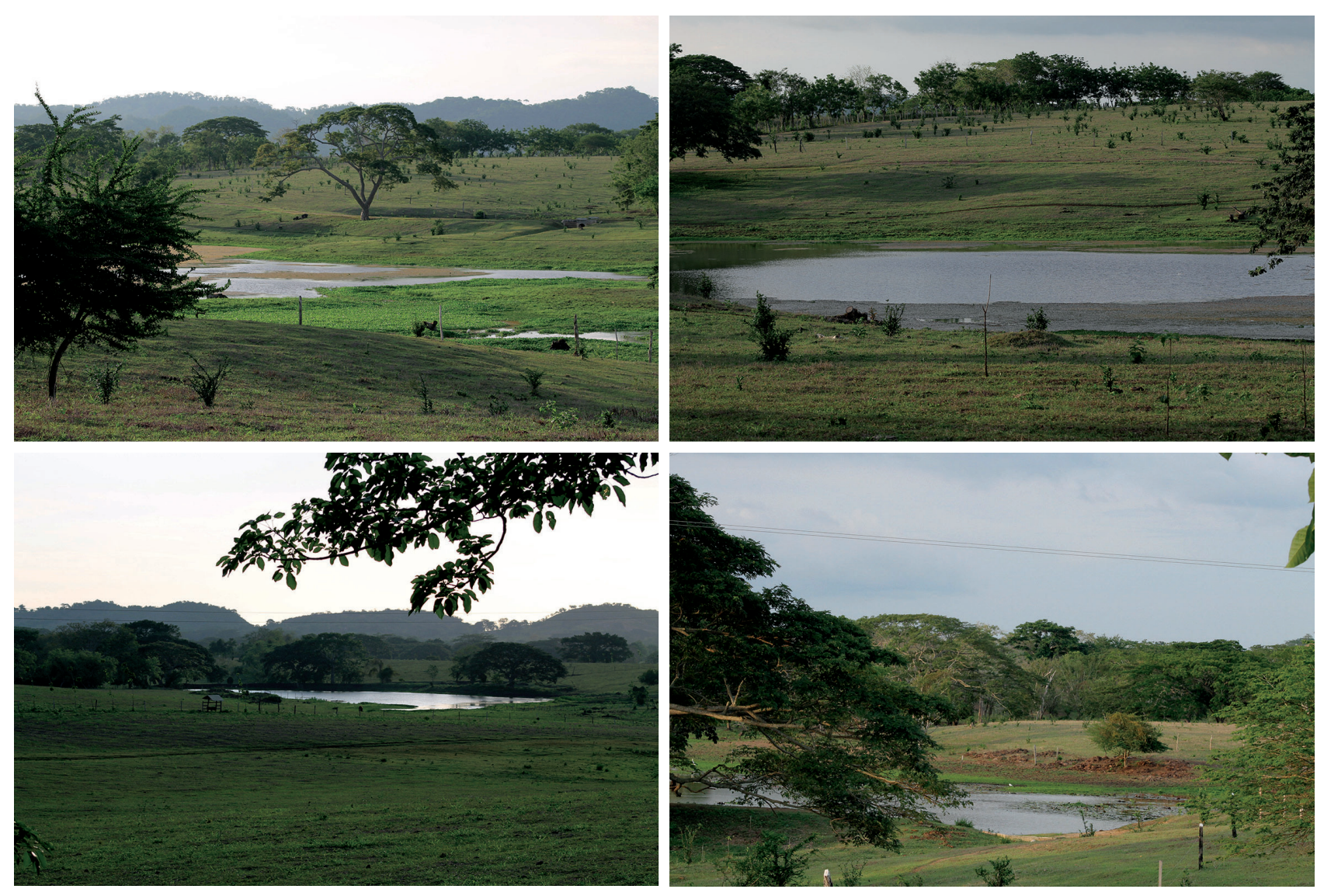

Figura 2. Algunos de los jagüeyes muestreados.

El cálculo de la población representativa de jagüeyes se obtuvo aplicando la matriz de tamaño de muestra para poblaciones finitas, con: $\mathrm{n}=65$ jagüeyes, $\mathrm{p}<0,05$, nivel de confianza (alfa) del $90 \%$, con error máximo de estimación del $10 \%$. Dio como resultado 12 unidades que fueron escogidas aleatoriamente (Zar 1999) (Tabla1).

Toma de información. Durante enero a marzo de 2014, correspondiente a la época de sequía, con horario de trabajo entre las 08:00 y las 12:00 horas, se llevó a cabo el trabajo de campo. Entre la sesión de captura y la de recaptura se tuvo un espacio de 30 días para cada uno de los jagüeyes. Se utilizó un trasmallo de $100 \mathrm{~m}$ de largo por $6 \mathrm{~m}$ de ancho y ojo de $5 \mathrm{~cm}$ (Vogt 1980, De La Ossa et al. 2012a). En cada sesión se hicieron dos barridos y se mantuvo un cubrimiento aproximado del $90 \%$ del área. Los animales capturados se identificaron a nivel de especie y fueron sexados, según lo establecido por Rueda-Almonacid et al. (2007).

Análisis de información poblacional. Se calculó media, máxima, minina y desviación estándar para las áreas de los jagüeyes; se aplicó la prueba de Wilcoxon para determinar diferencias entre tamaños poblaciones totales por especie (Zar 1999). Se calculó la densidad bruta indicada en ind./área (Krebs 1986).

Para el cálculo de la población por especie se recurrió a la aplicación del Método de Lincoln-Petersen, teniendo en cuenta que al momento del trabajo se trataba de una población cerrada que además se concentra por efectos climáticos, ya que la época de sequía impone un aislamiento de los jagüeyes y las condiciones de sequía generan un ambiente sumamente adverso que dificulta los movimientos poblacionales de quelonios (Gibbons et al. 1990). 
Tabla 1. Coordenadas de cada lugar de muestreo y medidas de área para cada jagüey.

\begin{tabular}{cccc}
\hline Jagüey & Área $\left(\mathbf{m}^{2}\right)$ & Longitud & Latitud \\
\hline 1 & $6.545,80$ & $75^{\circ} 32^{\prime} 20^{\prime \prime}$ & $9^{\circ} 33^{\prime} 0^{\prime \prime}$ \\
\hline 2 & $1.190,00$ & $75^{\circ} 32^{\prime} 31^{\prime \prime}$ & $9^{\circ} 33^{\prime} 47^{\prime \prime}$ \\
\hline 3 & $32.509,85$ & $7^{\circ} 33^{\prime} 29^{\prime \prime}$ & $9^{\circ} 43^{\prime} 12^{\prime \prime}$ \\
\hline 4 & $6.814,26$ & $75^{\circ} 35^{\prime} 10^{\prime \prime}$ & $9^{\circ} 39^{\prime} 58^{\prime \prime}$ \\
\hline 5 & $8.796,13$ & $75^{\circ} 30^{\prime} 22^{\prime \prime}$ & $9^{\circ} 36^{\prime} 14^{\prime \prime}$ \\
\hline 6 & $3.185,12$ & $75^{\circ} 31^{\prime} 37^{\prime \prime}$ & $9^{\circ} 37^{\prime} 48^{\prime \prime}$ \\
\hline 7 & $3.174,95$ & $75^{\circ} 35^{\prime} 35^{\prime \prime}$ & $9^{\circ} 41^{\prime} 24^{\prime \prime}$ \\
\hline 8 & $25.652,74$ & $75^{\circ} 31^{\prime} 41^{\prime \prime}$ & $9^{\circ} 43^{\prime} 30^{\prime \prime}$ \\
\hline 10 & $4.083,78$ & $75^{\circ} 33^{\prime} 7^{\circ}$ & $9^{\circ} 38^{\prime} 31^{\prime \prime}$ \\
\hline 11 & $1.172,37$ & $75^{\circ} 32^{\prime} 2^{\prime \prime}$ & $9^{\circ} 41^{\prime} 10^{\prime \prime}$ \\
\hline 12 & $2.831,50$ & $75^{\circ} 35^{\prime} 10^{\prime \prime}$ & $9^{\circ} 42^{\prime} 0^{\prime \prime}$ \\
\hline Área total & $97.344,67$ & & \\
\hline
\end{tabular}

Los animales fueron marcados para su futura identificación empleando el sistema de muescas sobre los escudos marginales y estableciendo un código de numeración (Rueda-Almonacid et al. 2007), luego fueron puestos en libertad en el mismo lugar de captura. Después de 30 días se extrajo una segunda muestra de animales entre los que había animales marcados. Asumiendo que la proporción de marcados en la segunda muestra es un razonable estimador de la proporción poblacional desconocida (Badii et al. 2012, Martella et al. 2012), se aplicó la siguiente fórmula.

$$
\mathrm{N}=\mathrm{M}(\mathrm{n}+1) / \mathrm{R}+1
$$

Donde: $\mathrm{N}=$ población a calcular; $\mathrm{M}=$ total de individuos capturados inicialmente; $\mathrm{N}=$ total de individuos recapturados; $\mathrm{R}=$ individuos recapturados que estaban marcados.

\section{Resultados}

Los 12 jagüeyes muestreados presentaron una media de $8.111,58 \mathrm{~m}^{2}$ (1.172-32.509, DE 10.187,08). El número de individuos capturados y recapturados se presenta en la tabla 2. Los datos de capturas y recapturas por especie se presentan en la tabla 2.

El número de individuos estimados por especie con un $95 \%$ de nivel de confianza fueron: T. callirostris 236 individuos (197 y 277); K. scorpiodes 68 individuos (45 y 95); M. dahli 77 individuos (53 y 104).

Al aplicar prueba de Wilcoxon para comparar entre las poblaciones totales de cada especie, se determinan diferencias entre T. callirostris con K. scorpioides y M. dahli (Tabla 3).

La densidad bruta total de acuerdo con la población estimada y calculada por especie se presenta en la tabla 4.

\section{Discusión}

Independientemente del área de los jagüeyes, en todos ellos se hallaron individuos de T. callirostris, $K$. scorpioides y $M$. dahli, lo que indica que estas especies hacen uso conjunto de este tipo de cuerpos de agua y probablemente son muy importantes para el mantenimiento de las poblaciones de las especies de tortugas continentales referidas. Es necesario reconocer que el deterioro del hábitat natural limita la capacidad de carga de los ecosistemas y hace que estrategias como la ocupación y colonización de hábitat antrópicos sean valiosos para la sobrevivencia de la especies locales (De La Ossa 1996, De La Ossa y De La Ossa-Lacayo 2013); los jagüeyes se convierten por lo tanto en refugios faunísticos de gran importancia regional (Botero et al. 2009).

La población estimada de $T$. callirostris es significativamente mayor que la de $K$. scorpioides y M. dahli, y no se determina diferencia significativa entre las poblaciones totales de estas dos últimas especies, lo cual coincide con lo reportado para jagüeyes de las sabanas de Sucre por Sampedro et al. (2012). 
Trachemys callirostris es el quelonio continental de mayor abundancia, lo cual concuerda con los resultados de este trabajo y con lo reportado para la región (De La Ossa 2003, Fuentes-Obeid et al. 2003, De La Ossa y De La Ossa-Lacayo 2011, De La OssaLacayo y De La Ossa 2012). No obstante, hay que resaltar que la zona de estudio no está conectada a grandes ciénagas ni a ríos, que se trata de sistemas de almacenamiento de agua de tipo antrópico, aislados y que solo actúan como refugios.
En cuanto a K. scorpiodes, ampliamente distribuida en una gran variedad de ambientes acuáticos permanentes, semipermanentes y temporales, usualmente prefiere aguas oscuras y pozos aislados de los cauces principales de los grandes ríos (Berry et al. 2012; Páez et al. 2012). Medem (1958, 1960) describió el hábitat de K. scorpioides en Suramérica (principalmente Colombia) como arroyos, pequeños pozos, lagos, pantanos y otros tipos de humedales.

Tabla 2. Capturas y recapturas para las tres especies de quelonios muestreadas.

\begin{tabular}{lcccc}
\hline & & \multicolumn{3}{c}{ Captura final } \\
\cline { 3 - 5 } Especie & $\begin{array}{c}\text { Captura } \\
\text { inicial }\end{array}$ & $\begin{array}{c}\text { Recaptura } \\
\text { marcados }\end{array}$ & $\begin{array}{c}\text { Captura no } \\
\text { marcados }\end{array}$ & Total \\
\hline T. callirostris & 124 & 61 & 56 & 117 \\
\hline K. scorpiodes & 28 & 15 & 23 & 38 \\
\hline M. dahli & 35 & 18 & 23 & 41 \\
\hline
\end{tabular}

Tabla 3. Prueba de Wilcoxon para comparar la población total muestreada para las tres especies de quelonios.

\begin{tabular}{lccc}
\hline \multicolumn{1}{c}{ Especies } & N & Z & p \\
\hline T. callirostris vs. K. scorpiodes & 12 & 3,059412 & 0,002218 \\
\hline T. callirostris vs. $M$. dahli & 12 & 2,934058 & 0,003346 \\
\hline K. scorpiodes vs. $M$. dahli & 12 & 1,325083 & 0,185145 \\
\hline
\end{tabular}

Tabla 4. Densidad bruta estimada por especie acorde con la población calculada según el método de Lincoln-Petersen.

\begin{tabular}{lccc}
\hline \multicolumn{1}{c}{ Especie } & $\begin{array}{c}\text { Población } \\
\text { calculada }\end{array}$ & Área total (ha) & $\begin{array}{c}\text { Densidad } \\
\text { (Ind./ha) }\end{array}$ \\
\hline T. callirostris & 236 & 97,34 & 2,424 \\
\hline K. scorpiodes & 68 & 97,34 & 0,698 \\
\hline M. dahli & 77 & 97,34 & 0,791 \\
\hline
\end{tabular}


Forero-Medina y Castaño-Mora (2011) encontraron un movimiento promedio durante dos días de $68 \mathrm{~m}$ (máximo $380 \mathrm{~m}$ ), y un desplazamiento neto promedio de $35 \mathrm{~m}$ (máximo $300 \mathrm{~m}$ ), lo que permite asumir que los jagüeyes además de ser un refugio son parte del hábitat colonizado y que ha estado ligados a la permanencia de esta especie. Quedaría por definir históricamente cómo eran sus poblaciones cuando la matriz paisajística no acusaba el deterioro que ahora existe y cuando los cuerpos de aguas predominantes eran naturales. Hay que tener en cuenta que se trata de una de las especie de quelonios de más ampliamente distribución en Colombia, con registros en casi todas la cuencas hidrográficas en el Caribe (Páez et al. 2012; Montes-Correa et al. 2014) .

Por su parte, para $M$. dahli, Rueda-Alamonacid et al. (2004) registraron entre ocho y 18 tortugas capturadas por kilometro de arroyo muestreado, con densidades de 20 a 60 tortugas capturadas/ha. En otros jagüeyes estos valores fueron de hasta 500 individuos capturados/ha. Por otro lado, Forero-Medina et al. (2011), para el departamento del Cesar, hallaron densidades entre 16 tortugas/ha y 170 tortugas/ha, por lo que la densidad de $0,63 \mathrm{ind} /$ ha hallado en este estudio es comparativamente muy baja. La ocupación de jagüeyes que esta especie hace está documentada y se le reconoce como habitante regular de los mismos (Sampedro et al. 2012).

El deterioro de hábitats naturales es la causa principal de la pérdida de la biodiversidad mundial, según lo señalado por Myers et al. (2000). Sin embargo, algunos agroecosistemas pueden conservar una parte sustancial de la biodiversidad de su anterior ecosistema natural y servir como zonas de amortiguación y complemento a las áreas protegidas o de reserva para la fauna silvestre local o migratoria (De La Ossa et al. 2012b).

Dado el cambio que acusa la matriz paisajística de la zona de estudio, lo jagüeyes actúan como valiosos refugios para las tres especies de quelonios continentales estudiados. En muchos casos, el jagüey es la única reserva de agua disponible para el ganado y para los pobladores locales durante la época de sequía y es una fuente de sobrevivencia de gran valor cultural y productivo (De La Ossa et al. 2012b), que muestra según lo aquí analizado su importancia en aspectos de conservación local de quelonios, así como de otras especie de fauna silvestre regional (Botero et al. 2009, De La Ossa et al. 2012b).

\section{Conclusiones}

Los jagüeyes, en este caso, se convierten en refugios de gran valor para los quelonios continentales, en especial al tratarse de una matriz paisajística que acusa un cambio estructural profundo.

Los jagüeyes muy a pesar de ser unidades creadas como abrevaderos de ganado, cumplen un papel muy importante en la conservación de fauna silvestre local y su protección debería ser una estrategia prioritaria.

Se deben continuar los trabajos investigativos en estas unidades de almacenamiento de agua, es posible que muchos grupos zoológicos subsistan localmente en virtud de su existencia y funcionalidad.

\section{Referencias}

Badii, M. H., A. Guillen, J. Landeros, E. Cerna, Y. Ochoa y J. Valenzuela. 2012. Muestreo por Métodos de CapturaRecaptura. Daena: International Journal of Good Conscience 7 (1): 97-131.

Berry, J. F., J. B. Iverson y G. Forero-Medina. Familias y especies de tortugas dulceacuícolas y terrestres de Colombia. Cap. 18. Pp. 340-349. En: Páez, V. P., M. A. Morales-Betancourt, C. A. Lasso, O. V. Castaño-Mora y B. C. Bock (Eds.). V. Biología y conservación de las tortugas continentales de Colombia. Serie Editorial Recursos Hidrobiológicos y Pesqueros Continentales de Colombia. Instituto de Investigación de Recursos Biológicos Alexander von Humboldt (IAvH). Bogotá, D. C., Colombia.

Botero, A. L., J. De La Ossa-V., A. Espitia y A. De La Ossa-Lacayo. 2009. Importancia de los jagüeyes en las sabanas del Caribe colombiano. Revista Colombiana de Ciencia Animal 1 (1): 71-84.

Casas, J. J., J. Toja, S. Bonachela, F. Fuentes, I. Gallego, M. Juan, D. León, P. Peñalver, C. Pérez y P. Sánchez. 2012a Artificial ponds in a Mediterranean region (Andalusia, southern Spain): agricultural and environmental issues. Water and Environment Journal 25: 308-317. 
Castaño-M., O. V. (Ed.). 2002. Libro rojo de reptiles de Colombia. Instituto de Ciencias Naturales, Universidad Nacional de Colombia, Ministerio de Medio Ambiente, Conservación Internacional-Colombia. Bogotá. 160 pp.

CITES. 2016. Appendices I, II, and III. Disponible en: https://cites.org/sites/default/files/eng/app/2016/SAppendices-2016-03-10.pdf. consultado: 10-01-2017.

De La Ossa V., J. 2003. Manejo de fauna silvestre tropical. Pp. 29-34. Programa de Desarrollo Sostenible de la Región de La Mojana. DNP, FAO, Bogotá, Colombia.

De La Ossa-V., J., G. Cárdenas-Arévalo y V. P. Páez. 2012a. Métodos de campo para estudios demográficos. Capítulo 13. Pp. 171-186. En: Páez, V. P., M. A. Morales-Betancourt, C. A. Lasso, O. V. Castaño-Mora y B. C. Bock (Eds). Biología y conservación de las tortugas continentales de Colombia. Serie Editorial Recursos Hidrobiológicos y Pesqueros Continentales de Colombia. Instituto de Investigación de Recursos Biológicos Alexander von Humboldt (IAvH). Bogotá, D. C., Colombia.

De La Ossa-V., J., S. Galván-Guevara y A. De La OssaLacayo. 2102b. Importancia del jagüey ganadero en la conservación local de aves silvestres en el Caribe colombiano. Revista U.D.C.A Actualidad \& Divulgación Cientifica 15 (1): $181-186$.

De La Ossa-V., J. y A. De La Ossa-Lacayo. 2011. Cacería de subsistencia en San Marcos, Sucre, Colombia. Revista Colombiana de Ciencia Animal 3 (2): 213-224.

De La Ossa-Lacayo, A. y J. De La Ossa-V. 2012. Utilización de fauna silvestre en el área rural de Caimito, Sucre, Colombia. Revista Colombiana de Ciencia Animal 4 (1): 46-58.

De La Ossa-Lacayo, A. 2014. Jagüeyes: ecosistemas lénticos y antrópicos como alternativa para la conservación de Caiman crocodilus fuscus (Crocodylia: Alligatoridae) en el golfo de Morrosquillo, Sucre, Colombia. Revista de la Asociación Colombiana de Ciencias Biológicas 26: 21-28.

De La Ossa-V., J. y A. De La Ossa-Lacayo. 2013. Ocupación de jagüeyes por la babilla, Caiman crocodilus fuscus (Cope, 1868), en el Caribe colombiano. Biota Colombiana 14 (2): 327-336.

Forero-Medina., G., G. Cárdenas-Arévalo y O. V. Castaño-Mora. 2011. Abundance, home range, and movement patterns of the endemic species Dahl's toadheaded turtle (Mesoclemmys dahli) in Cesar, Colombia. Chelonian Conservation and Biology 10: 228-236.

Forero-Medina, G. y O. V. Castaño-Mora. 2011. Kinosternon scorpioides albogulare (Dumeril and Bocourt 1870) - white throated mud turtle, swanka turtle. Chelonian Research Monographs 5: 064.1-064.5.

Fuentes-Obeid, S., A. Sampedro y M. Ardila-Marulanda. 2003. Importancia de la jicotea (Trachemys scripta callirostris: Chelonia, Emydidae) como recurso natural en la comunidad de isla del Coco, Región de La Mojana, Departamento de Sucre, Colombia. Revista Biología 17 (2): 126-133.

Gibbons, J. W., J. L. Greene y J. D. Congdon. 1990. Temporal and spatial movement patterns of sliders and other turtles. Pp. 201-215. En: Gibbons, J. W. (Ed.). Life history and ecology of the Slider Turtle. Smithsonian Institution Press. Washington, DC.

Hernández-Camacho, J. y H. Sánchez-Páez. 1992. Biomas terrestres de Colombia. Pp. 153-173. En: Halffter, G. (Ed). La diversidad biológica iberoamericana I. Acta Zoológica Mexicana. México.

Holdridge, L. R. 1967. Life Zone Ecology. Tropical Science Center. San José, Costa Rica. 1a. ed. San José, Costa Rica. 149 pp.

Krebs, C. J. 1986. Ecología. Ediciones Pirámide. Madrid, España. 172 pp.

León, D., P. Peñalver, J. J. Casas, M. Juan, F. FuentesRodríguez, I. Gallego y J. Toja. 2010. Zooplankton richness in farm ponds of Andalusia (southern Spain). A comparison with natural wetlands. Limnetica 29: 153-162.

Martella, B. M., E. Trumper, M. L. Bellis, D. Renison, F. P. Giordano, G. Bazzano y M. R. Gleiser. 2012. Manual de Ecología Poblaciones: Introducción a las técnicas para el estudio de las poblaciones silvestres. Biología, Serie Ecología 5 (1): 1-31.

Medem, F. 1958. Informe sobre reptiles colombianos (II). El conocimiento actual sobre la distribución geográfica de las Testudinata en Colombia. Boletín del Museo de Ciencias Naturales, Caracas 2-3: 13-45.

Medem, F. 1960. Datos zoogeográficos y ecológicos sobre los Crocodylia y Testudinata de los ríos Amazonas, Putumayo y Caquetá. Caldasia 8: 341-351.

Medem, F. 1962. La distribución geográfica y ecológica de los Crocodylia y Testudinata en el departamento del Chocó. Revista de la Academia Colombiana de Ciencias Exactas, Físicas y Naturales 11 (44): 279-342

Medem, F. 1966. Contribuciones al conocimiento sobre la ecología y distribución geográfica de Phrynops (Batrachemys) dahli; (Testudinata, Pleurodira, Chelidae). Caldasia 9 (3): 467-482.

Montes-Correa, A. C., L. P. Saboyá-Acosta, V. Páez, K. Veja y J. M. Renjifo. 2014. Distribución de tortugas continentales del Caribe colombiano. Acta Biologica Colombiana 19 (3): 341-350.

Morales-Betancourt, M. A., C. A. Lasso, V. P. Páez y B. C. Bock. 2015. Libro rojo de reptiles de Colombia. 2015. Instituto de Investigación de Recursos Biológicos Alexander von Humboldt (IAvH), Universidad de Antioquia. Bogotá, D. C., Colombia. 258 pp.

Myers, N., R. A. Mittermeier, C. G. Mittermeier, G. A. B. 
da Fonseca y J. Kent. 2000. Biodiversity hotspots for conservation priorities. Nature 403: 853-858.

Páez, V. P., M. A. Morales-Betancourt, C. A. Lasso, O. V. Castaño-Mora y B. C. Bock (Eds.). 2012. V. Biología y conservación de las tortugas continentales de Colombia. Serie Editorial Recursos Hidrobiológicos y Pesqueros Continentales de Colombia. Instituto de Investigación de Recursos Biológicos Alexander von Humboldt (IAvH). Bogotá, D. C., Colombia, 528 pp.

Rueda-Almonacid, J. V. M, J. L. Carr, R. A. Mittermeier, J. V. Rodríguez-Mahecha, R. B. Mast, R. C. Vogt, A. G. J. Rhodin, J. De La Ossa-Velásquez, J. N. Rueda y C. G. Mittermeier. 2007. Las tortugas y cocodrilos de los países andinos del trópico. Serie de guías tropicales de campo. Conservación Internacional. Bogotá, Colombia. 270 pp.

Rueda-A., J. V., P. A. Galvis, C. López y G. Y. Lozano. 2004. Estudio sobre la distribución geográfica y el estatus ecológico de la tortuga carranchina (Batrachemys dahli) en el bajo Sinú, departamento de Córdoba. Conservación Internacional, Corporación Autónoma Regional de los Valles del Sinú y San Jorge. Diagnóstico y acciones de conservación de especies acuáticas amenazadas en la ecoregión del bajo Sinú. Informe final CI-CVS, Colombia. 53 pp.

Sampedro-Marín, A., P. Tobíos-Atencio y T. TrespalacioSolana, T. 2012. Estado de conservación de la tortuga "carranchina" (Batrachemys dahli) en localidades del departamento de Sucre, Colombia. Revista Colombiana de Ciencia Animal 4 (1): 69-88.

Sancho, V. e I. Lacomba. 2010. Conservación y restauración de puntos de agua para la biodiversidad. Colección Manuales Técnicos de Biodiversidad, 2. Generalitat. Conselleria de Medi Ambient, Aigua, Urbanisme i Habitage. 73 pp.

Verdiell-Cubedo, D. 2012. Las charcas ganaderas en la región de Murcia: hábitats acuáticos relevantes para la conservación de la biodiversidad regional. Revista Eubacteria 29:1-4.

Vogt, R. C. 1980. New methods for trapping aquatic turtles. Copeia 1980: 368-371.

Zar, J. H. 1999. Biostatistical Analysis, 4th. Ed. Prentice Hall, Upper Saddle River, NJ. USA. 662 pp.

\section{Jaime De La Ossa-V.}

Grupo de Investigación en Biodiversidad Tropical, Facultad de Ciencias Agropecuarias,

Universidad de Sucre, Sincelejo (Sucre), Colombia jaimedelaossa@yahoo.com

Merly Ardila-Marulanda

Universidad de Sucre, Sincelejo (Sucre), Colombia merlyam@yahoo.es

\section{Alejandro De La Ossa-Lacayo}

Selvagua SAS, Grupo de Investigación en Biodiversidad Tropical, Universidad de Sucre, Sincelejo (Sucre), Colombia

alejandrodelaossa@yahoo.com
Jagüeyes y su papel potencial en la conservación de tortugas continentales en el golfo de Morrosquillo, Sucre, Caribe colombiano

Citación del artículo: De La Ossa-V., J., M. Ardila-Marulanda y A. De La Ossa-Lacayo. 2017. Jagüeyes y su papel potencial en la conservación de tortugas continentales en el golfo de Morrosquillo, Sucre, Caribe colombiano. Biota Colombiana 18 (1): 316-324. DOI: 10.21068/c2017.v18n01a18.

Recibido: 5 de octubre de 2016

Aprobado: 9 de marzo de 2017 


\section{Guía para autores}

(humboldt.org.co/es/bibliotecaypublicaciones/biota)

\section{Preparación del manuscrito}

El envío de un manuscrito implica la declaración explícita por parte del autor(es) de que este no ha sido previamente publicado, ni aceptado para su publicación en otra revista u otro órgano de difusión científica. Todas las contribuciones son de la entera responsabilidad de sus autores y no del Instituto de Investigación de Recursos Biológicos Alexander von Humboldt, ni de la revista o sus editores.

Los trabajos pueden estar escritos en español, inglés o portugués, y se recomienda que no excedan las 40 páginas (párrafo espaciado a 1,5 líneas) incluyendo tablas, figuras y anexos. En casos especiales el editor podrá considerar la publicación de trabajos más extensos, monografías o actas de congresos, talleres o simposios. De particular interés para la revista son las descripciones de especies nuevas para la ciencia, nuevos registros geográficos y listados de la biodiversidad regional.

Para la elaboración de los textos del manuscrito se puede usar cualquier procesador de palabras (preferiblemente Word); los listados (a manera de tabla) deben ser elaborados en una hoja de cálculo (preferiblemente Excel). Para someter un manuscrito es necesario además anexar una carta de intención en la que se indique claramente:

1. Nombre completo del (los) autor (es), y direcciones para envío de correspondencia (es indispensable suministrar una dirección de correo electrónico para comunicación directa).

\section{Título completo del manuscrito.}

3. Nombres, tamaños y tipos de archivos suministrados.

4. Lista mínimo de tres revisores sugeridos que puedan evaluar el manuscrito, con sus respectivas direcciones electrónicas.

\section{Evaluación del manuscrito}

Los manuscritos sometidos serán revisados por pares científicos calificados, cuya respuesta final de evaluación puede ser: a) aceptado (en cuyo caso se asume que no existe ningún cambio, omisión o adición al artículo, y que se recomienda su publicación en la forma actualmente presentada); b) aceptación condicional (se acepta y recomienda el artículo para su publicación solo si se realizan los cambios indicados por el evaluador); y c) rechazo (cuando el evaluador considera que los contenidos o forma de presentación del artículo no se ajustan a los requerimientos y estándares de calidad de Biota Colombiana).

\section{Texto}

- Para la presentación del manuscrito configure las páginas de la siguiente manera: hoja tamaño carta, márgenes de $2,5 \mathrm{~cm}$ en todos los lados, interlineado 1,5 y alineación hacia la izquierda (incluyendo título y bibliografía).

- Todas las páginas de texto (a excepción de la primera correspondiente al título), deben numerarse en la parte inferior derecha de la hoja.
- Use letra Times New Roman o Arial, tamaño 12 puntos en todos los textos. Máximo 40 páginas, incluyendo tablas, figuras y anexos. Para tablas cambie el tamaño de la fuente a 10 puntos. Evite el uso de negritas o subrayados.

- Los manuscritos debe llevar el siguiente orden: título, resumen y palabras clave, abstract y key words, introducción, material y métodos, resultados, discusión, conclusiones (optativo), agradecimientos (optativo) y bibliografía. Seguidamente, presente una página con la lista de tablas, figuras y anexos. Finalmente, incluya las tablas, figuras y anexos en archivos separadas, debidamente identificadas.

- Escriba los nombres científicos de géneros, especies y subespecies en cursiva (itálica). Proceda de la misma forma con los términos en latín (p. e. sensu, et al.). No subraye ninguna otra palabra o título. No utilice notas al pie de página.

- En cuanto a las abreviaturas y sistema métrico decimal, utilice las normas del Sistema Internacional de Unidades (SI) recordando que siempre se debe dejar un espacio libre entre el valor numérico y la unidad de medida (p. e. $16 \mathrm{~km}, 23^{\circ} \mathrm{C}$ ). Para medidas relativas como m/seg., use m.seg ${ }^{-1}$.

- Escriba los números del uno al diez siempre con letras, excepto cuando preceden a una unidad de medida (p. e. $9 \mathrm{~cm}$ ) o si se utilizan como marcadores (p. e. parcela 2, muestra 7).

- No utilice punto para separar los millares, millones, etc. Utilice la coma para separar en la cifra la parte entera de la decimal (p. e. 3,1416). Enumere las horas del día de 0:00 a 24:00.

- Exprese los años con todas las cifras sin demarcadores de miles (p. e. 1996-1998). En español los nombres de los meses y días (enero, julio, sábado, lunes) siempre se escriben con la primera letra minúscula, no así en inglés.

- Los puntos cardinales (norte, sur, este y oeste) siempre deben ser escritos en minúscula, a excepción de sus abreviaturas $\mathrm{N}, \mathrm{S}$, $\mathrm{E}, \mathrm{O}$ (en inglés W), etc. La indicación correcta de coordenadas geográficas es como sigue: $02^{\circ} 37^{\prime} 53^{\prime \prime} \mathrm{N}-56^{\circ} 28^{\prime} 53^{\prime \prime} \mathrm{O}$. La altitud geográfica se citará como se expresa a continuación: 1180 m s.n.m. (en inglés $1180 \mathrm{~m}$ a.s.l).

- Las abreviaturas se explican únicamente la primera vez que son usadas.

- Al citar las referencias en el texto mencione los apellidos de los autores en caso de que sean uno o dos, y el apellido del primero seguido por et al. cuando sean tres o más. Si menciona varias referencias, éstas deben ser ordenadas cronológicamente y separadas por comas (p. e. Rojas 1978, Bailey et al. 1983, Sephton 2001, 2001).

- Resumen: incluya un resumen de máximo 200 palabras, tanto en español o portugués como inglés.

- Palabras Clave: máximo seis palabras clave, preferiblemente complementarias al título del artículo, en español e inglés. 


\section{Agradecimientos}

Opcional. Párrafo sencillo y conciso entre el texto y la bibliografía. Evite títulos como Dr., Lic., TSU, etc.

\section{Fotografías, figuras, tablas y anexos}

Refiera las figuras (gráficas, diagramas, ilustraciones y fotografías) sin abreviación (p. e. Figura 3) al igual que las tablas (p. e. Tabla 1). Gráficos (p. e. CPUE anuales) y figuras (histogramas de tallas), preferiblemente en blanco y negro, con tipo y tamaño de letra uniforme. Deben ser nítidas y de buena calidad, evitando complejidades innecesarias (por ejemplo, tridimensionalidad en gráficos de barras); cuando sea posible use solo colores sólidos en lugar de tramas. Las letras, números o símbolos de las figuras deben ser de un tamaño adecuado de manera que sean claramente legibles una vez reducidas. Para el caso de las fotografías y figuras digitales es necesario que estas sean guardadas como formato tiff con una resolución de 300 dpi. Es oportuno que indique en qué parte del texto desea insertarla.

Lo mismo aplica para las tablas y anexos, los cuales deben ser simples en su estructura (marcos) y estar unificados. Presente las tablas en archivo aparte (Excel), identificadas con su respectivo número. Haga las llamadas a pie de página de tabla con letras ubicadas como superíndice. Evite tablas grandes sobrecargadas de información y líneas divisorias o presentadas en forma compleja. Es oportuno que indique en qué parte del texto desea insertar tablas y anexos.

\section{Bibliografía}

Contiene únicamente la lista de las referencias citadas en el texto. Ordénelas alfabéticamente por autores y cronológicamente para un mismo autor. Si hay varias referencias de un mismo autor(es) en el mismo año, añada las letras a, b, c, etc. No abrevie los nombres de las revistas. Presente las referencias en el formato anexo, incluyendo el uso de espacios, comas, puntos, mayúsculas, etc.

\section{Artículo EN REVISTAS}

Agosti, D., C. R. Brandao y S. Diniz. 1999. The new world species of the subfamily Leptanilloidinae (Hymenoptera: Formicidae). Systematic Entomology 24: 14-20.

\section{LIBROS, TESIS E INFORMES TÉCNICOS}

Libros: Gutiérrez, F. P. 2010. Los recursos hidrobiológicos y pesqueros en Colombia. Instituto de Investigación de Recursos Biológicos Alexander von Humboldt. Bogotá, D. C., 118 pp.

Tesis: Cipamocha, C. A. 2002. Caracterización de especies y evaluación trófica de la subienda de peces en el raudal Chorro de Córdoba, bajo río Caquetá, Amazonas, Colombia. Trabajo de grado. Universidad Nacional de Colombia, Facultad de Ciencias, Departamento de Biología. Bogotá D. C., 160 pp.

Informes técnicos: Andrade, G. I. 2010. Gestión del conocimiento para la gestión de la biodiversidad: bases conceptuales y propuesta programática para la reingeniería del Instituto Humboldt. Informe Técnico. Instituto de Investigación de Recursos Biológicos Alexander von Humboldt. Bogotá D. C., 80 pp.

Capítulo en libro o en informe: Fernández F., E. E. Palacio y W. P. MacKay. 1996. Introducción al estudio de las hormigas (Hymenoptera: Formicidae) de Colombia. Pp:349-412. En: Amat, G. D., G. Andrade y F. Fernández (Eds.). Insectos de Colombia. Estudios Escogidos. Academia Colombiana de Ciencias Exactas, Físicas y Naturales \& Centro Editorial Javeriano, Bogotá.

Resumen en congreso, simposio, talleres: Señaris, J. C. 2001. Distribución geográfica y utilización del hábitat de las ranas de cristal (Anura; Centrolenidae) en Venezuela. En: Programa y Libro de Resúmenes del IV Congreso Venezolano de Ecología. Mérida, Venezuela, p. 124.

PÁginas Web

No serán incluidas en la bibliografía, sino que se señalarán claramente en el texto al momento de mencionarlas.

\title{
Guidelines for authors
}

\author{
(humboldt.org.co/es/bibliotecaypublicaciones/biota)
}

\section{Manuscript preparation}

Submitting a manuscript implies the explicit statement by the author(s) that the paper has not been published before nor accepted for publication in another journal or other means of scientific diffusion. Contributions are entire responsibility of the author and not the Alexander von Humboldt Institute for Research on Biological Resources, or the journal and their editors.

Papers can be written in Spanish, English or Portuguese and it is recommended not exceeding 40 pages (with paragraphs spaced at 1,5) including tables, figures and Annex. For special cases, the editor could consider publishing more extensive papers, monographs or symposium conclusions. New species descriptions for science, new geographic records and regional biodiversity lists are of particular interest for this journal.
Any word-processor program may be used for the text (Word is recommended). taxonomic list or any other type of table, should be prepared in spreadsheet aplication (Excel is recommended). To submit a manuscript must be accompanied by a cover letter which clearly indicate $\mathrm{s}$ :

1. Full names, mailing addresses and e-mail addresses of all authors. (Please note that email addresses are essential to direct communication).

2. The complete title of the article.

3. Names, sizes, and types of files provide.

4. A list of the names and addresses of at least three (3) reviewers who are qualified to evaluate the manuscript. 


\section{Evaluation}

Submitted manuscript will have a peer review evaluation. Resulting in any of the following: a) accepted (in this case we assume that no change, omission or addition to the article is required and it will be published as presented.); b) conditional acceptance (the article is accepted and recommended to be published but it needs to be corrected as indicated by the reviewer); and c) rejected (when the reviewer considers that the contents and/or form of the paper are not in accordance with requirements of publication standards of Biota Colombiana).

\section{Text}

- The manuscript specifications should be the following: standard letter size paper, with $2.5 \mathrm{~cm}$ margins on all sides, 1.5-spaced and left-aligned (including title and bibliography).

- All text pages (with the exception of the title page) should be numbered. Pages should be numbered in the lower right corner.

- Use Times New Roman or Arial font, size 12, for all texts. Use size 10 text in tables. Avoid the use of bold or underlining. 40 pages maximum, including tables, figures and annex. For tables use size 10 Times New Roman or Arial Font (the one used earlier).

- The manuscripts must be completed with the following order: title, abstract and key words, then in Spanish Título, Resumen y Palabras claves. Introduction, Materials and Methods, Results, Discussion, conclusions (optional), acknowledgements (optional) and bibliography. Following include a page with the Table, Figure and Annex list. Finally tables, figures and annex should be presented and clearly identified in separate tables.

- Scientific names of genera, species and subspecies should be written in italic. The same goes for Latin technical terms (i.e sensu, et al.). Avoid the use of underlining any word or title. Do not use footnotes.

- As for abbreviations and the metric system, use the standards of the International System of Units (SI) remembering that there should always be a space between the numeric value and the measure unit (e.g., $16 \mathrm{~km}, 23{ }^{\circ} \mathrm{C}$ ). For relative measures such as $\mathrm{m} / \mathrm{sec}$, use $\mathrm{m} \cdot \mathrm{sec}^{-1}$.

- Write out numbers between one to ten in letters except when it precedes a measure unit (e.g., $9 \mathrm{~cm}$ ) or if it is used as a marker (e.g., lot 9, sample 7).

- Do not use a point to seperate thousands, millions, etc. Use a comma to separate the whole part of the decimal (e.g., 3,1416). Numerate the hours of the from 0:00 to 24:00. Express years with all numbers and without marking thousands (e.g., 19961998). In Spanish, the names of the months and days (enero, julio, sábado, lunes) are always written with the first letter as a lower case, but it is not this way in English.

- The cardinal points (north, south, east, and west) should always be written in lower case, with the excpetino of abbreviations $\mathrm{N}, \mathrm{S}, \mathrm{E}, \mathrm{O}$ (in English NW), etc. The correct indication of geographic coordinates is as follows: $02^{\circ} 37^{\prime} 53^{\prime \prime} \mathrm{N}-56^{\circ} 28^{\prime} 53^{\prime \prime} \mathrm{O}$. The geographic altitude should be cited as follows: $1180 \mathrm{~m}$ a.s.l.

- Abbreviations are explained only the first time they are used.
- When quoting references in the text mentioned author's last names when they are one or two, and et al. after the last name of the first author when there are three or more. If you mention many references, they should be in chronological order and separated by commas (e.g., Rojas 1978, Bailey et al. 1983, Sephton 2001, 2001).

- ABSTRACT: include an abstract of 200 words maximum, in Spanish, Portuguese or English.

- KeY WORDS: six key words maximum, complementary to the title.

\section{Pictures, Figures, Tables and Annex}

- Figures (graphics, diagrams, illustrations and photographs) without abbreviation (e.g. Figure 3) the same as tables (e.g., Table 1). Graphics and figures should be in black and white, with uniform font type and size. They should be sharp and of good quality, avoiding unnecessary complexities (e.g., three dimensions graphics). When possible use solid color instead of other schemes. The words, numbers or symbols of figures should be of an adequate size so they are readable once reduced. Digital figures must be sent at 300 dpi and in .tiff format. Please indicate in which part of the text you would like to include it.

- The same applies to tables and annexes, which should be simple in structure (frames) and be unified. Present tables in a separate file (Excel), identified with their respective number. Make calls to table footnotes with superscript letters above. Avoid large tables of information overload and fault lines or presented in a complex way. It is appropriate to indicate where in the text to insert tables and annexes.

\section{Bibliography}

References in bibliography contains only the list of references cited in the text. Sort them alphabetically by authors and chronologically by the same author. If there are several references by the same author(s) in the same year, add letters a, b, c, etc. Do not abbreviate journal names. Present references in the attached format, including the use of spaces, commas, periodss, capital letters, etc.

\section{JOURNAL ARTICLE}

Agosti, D., C. R. Brandao y S. Diniz. 1999. The new world species of the subfamily Leptanilloidinae (Hymenoptera: Formicidae). Systematic Entomology 24: 14-20.

\section{BOOK, THESIS, TECHNICAL REVIEWS}

Book: Gutiérrez, F. P. 2010. Los recursos hidrobiológicos y pesqueros en Colombia. Instituto de Investigación de Recursos Biológicos Alexander von Humboldt. Bogotá, D. C. 118 pp.

Thesis: Cipamocha, C. A. 2002. Caracterización de especies y evaluación trófica de la subienda de peces en el raudal Chorro de Córdoba, bajo río Caquetá, Amazonas, Colombia. Trabajo de grado. Universidad Nacional de Colombia, Facultad de Ciencias, Departamento de Biología. Bogotá D. C. 160 pp.

Technical reviews: Andrade, G. I. 2010. Gestión del conocimiento para la gestión de la biodiversidad: bases conceptuales y propuesta programática para la reingeniería del Instituto Humboldt. Informe 
Técnico. Instituto de Investigación de Recursos Biológicos Alexander von Humboldt. Bogotá D. C. 80 pp.

Book chapter or in review: Fernández F., E. E. Palacio y W. P. MacKay. 1996. Introducción al estudio de las hormigas (Hymenoptera: Formicidae) de Colombia. Pp: 349-412. En: Amat, G. D., G. Andrade y F. Fernández (Eds.). Insectos de Colombia. Estudios Escogidos. Academia Colombiana de Ciencias Exactas, Físicas y Naturales \& Centro Editorial Javeriano, Bogotá.
Symposium abstract: Señaris, J. C. 2001. Distribución geográfica y utilización del hábitat de las ranas de cristal (Anura; Centrolenidae) en Venezuela. En: Programa y Libro de Resúmenes del IV Congreso Venezolano de Ecología. Mérida, Venezuela, p. 124.

WeB PAGES

Not be included in the literature, but clearly identified in the text at the time of mention.

\section{Guía para autores - Artículos de Datos}

www.humboldt.org.co/es/bibliotecaypublicaciones/biota- biotacol@humboldt.org.co www.sibcolombia.net - sib+iac@humboldt.org.co

El objetivo de esta guía es establecer y explicar los pasos necesarios para la elaboración de un manuscrito con el potencial de convertirse en artículo de datos para ser publicado en la revista Biota Colombiana. En esta guía se incluyen aspectos relacionados con la preparación de datos y el manuscrito.

\section{¿Qué es un artículo de datos?}

Un artículo de datos o Data Paper es un tipo de publicación académica que ha surgido como mecanismo para incentivar la publicación de datos sobre biodiversidad, a la vez que es un medio para generar reconocimiento académico y profesional adecuado a todas las personas que intervienen de una manera u otra en la gestión de información sobre biodiversidad.

Los artículos de datos contienen las secciones básicas de un artículo científico tradicional. Sin embargo, estas se estructuran de acuerdo a un estándar internacional para metadatos (información que le da contexto a los datos) conocido como el GBIF Metadata Profile (GMP) ${ }^{1}$. La estructuración del manuscrito con base en este estándar se da, en primer lugar, para facilitar que la comunidad de autores que publican conjuntos de datos a nivel global, con presencia en redes como la Global Biodiversity Information Facility (GBIF) y otras redes relacionadas, puedan publicar fácilmente artículos de datos obteniendo el reconocimiento adecuado a su labor. En segundo lugar, para estimular que los autores de este tipo de conjuntos de datos que aún no han publicado en estas redes de información global, tengan los estímulos necesarios para hacerlo.

Un artículo de datos debe describir de la mejor manera posible el quién, qué, dónde, cuándo, por qué y cómo de la toma y almacenamiento de los datos, sin llegar a convertirse en el medio para realizar un análisis exhaustivo de los mismos, como sucede en otro tipo de publicaciones académicas. Para profundizar en este modelo de publicación se recomienda consultar a Chavan y Penev $(2011)^{2}$.

\section{¿Qué manuscritos pueden llegar a ser artículos de datos?}

Manuscritos que describan conjuntos de datos primarios y originales que contengan registros biológicos (captura de datos de la presencia de un(os) organismo(s) en un lugar y tiempo determinados); información asociada a ejemplares de colecciones biológicas; listados temáticos o geográficos de especies; datos genómicos y todos aquellos datos que sean susceptibles de ser

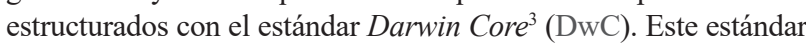
es utilizado dentro de la comunidad de autores que publican conjuntos de datos sobre biodiversidad para estructurar los datos y de esta manera poder consolidarlos e integrarlos desde diferentes fuentes a nivel global. No se recomienda someter manuscritos que describan conjuntos de datos secundarios, como por ejemplo compilaciones de registros biológicos desde fuentes secundarias (p.e. literatura o compilaciones de registros ya publicados en redes como GBIF o IABIN).

\section{Preparación de los datos}

Como se mencionó anteriormente los datos sometidos dentro de este proceso deben ser estructurados en el estándar DwC. Para facilitar su estructuración, el Sistema de Información sobre Biodiversidad de Colombia (SiB Colombia), ha creado dos plantillas en Excel, una para registros biológicos y otra para listas de especies. Lea y siga detenidamente las instrucciones de las plantillas para la estructuración de los datos a publicar. Para cualquier duda sobre el proceso de estructuración de estos datos por favor contactar al equipo coordinador del SiB Colombia (ECSiB) en sib+iac@humboldt.org.co.

\footnotetext{
${ }^{1}$ Wieczorek, J. 2011. Perfil de Metadatos de GBIF: una guía de referencia rápida. En: Wieczorek, J. The GBIF Integrated Publishing Toolkit User Manual, version 2.0. Traducido y adaptado del inglés por D. Escobar. Sistema de Información sobre Biodiversidad de Colombia, Bogotá D.C., Colombia, 23p. Disponible en http://www.sibcolombia.net/repositorio-de-documentos.

${ }^{2}$ Chavan, V. y L. Penev. 2011. The data paper: The mechanism to incentivize data publishing in biodiversity science. BMC Bioinformatics 12 (Suppl 15): S2.

${ }^{3}$ TDWG. 2011. Darwin Core: una guía de referencia rápida. (Versión original producida por TDWG, traducida al idioma español por Escobar, D.; versión 2.0). Bogotá: SiB Colombia, 33 pp. Disponible en http://www.sibcolombia.net/repositorio-de-documentos
} 


\section{Preparación del manuscrito}

Para facilitar la creación y estructuración del manuscrito en el estándar GMP, se cuenta con la ayuda de un editor electrónico (http://ipt.sibcolombia.net/biota) que guiará al autor en dicho proceso y que finalmente generará una primera versión del manuscrito. Se recomienda el uso del manual GMP, como una guía de la información a incluir en cada sección del manuscrito, junto con el anexo 1 .

Pasos a seguir para la elaboración del manuscrito:

1 Solicite al correo sib+iac@humboldt.org.co el acceso al editor electrónico. El EC-SiB le asignará un usuario y contraseña.

2. Ingrese con su usuario y contraseña al editor electrónico, luego diríjase a la pestaña Gestión de recursos y cree un nuevo recurso asignando un nombre corto a su manuscrito usando el formato "AcrónimoDeLaInstitución_año_tipoDeConjuntoDeDatos", p.e. ABC_2010_avestinije y dar clic en el botón crear.

3. En la vista general del editor seleccione "editar" en la pestaña Metadatos (por favor, no manipule ningún otro elemento), allí encontrará diferentes secciones (panel derecho) que lo guiarán en la creación de su manuscrito. Guarde los cambios al finalizar cada sección, de lo contrario perderá la información. Recuerde usar el manual GMP. A continuación se presentan algunas recomendaciones para la construcción del manuscrito. Las secciones se indican en MAYUSCULAS y los elementos de dichas secciones en negrilla.

- En PARTES ASOCIADAS incluya únicamente aquellas personas que no haya incluido en INFORMACIÓN BÁSICA.

- Los DATOS DEL PROYECTO y DATOS DE LA COLECCIÓN son opcionales según el tipo de datos. En caso de usar dichas secciones amplíe o complemente información ya suministrada, p. ej. no repita información de la descripción (COBERTURA GEOGRÁFICA) en la descripción del área de estudio (DATOS DEL PROYECTO).

- De igual manera, en los MÉTODOS DE MUESTREO, debe ampliar o complementar información, no repetirla. La información del área de estudio debe dar un contexto específico a la metodología de muestreo.

- Es indispensable documentar el control de calidad en MÉTODOS DE MUESTREO. Acá se debe describir que herramientas o protocolos se utilizaron para garantizar la calidad y coherencia de los datos estructurados con el estándar DwC.

- Para crear la referencia del recurso, en la sección REFERENCIAS, utilice uno de los dos formatos propuestos (Anexo 2). No llene el identificador de la referencia, este será suministrado posteriormente por el EC-SiB.

- Para incluir la bibliografía del manuscrito en referencias, ingrese cada una de las citas de manera individual, añadiendo una nueva referencia cada vez haciendo clic en la esquina inferior izquierda.

4. Rectifique que el formato de la información suministrada cumpla con los lineamientos de la revista (p. ej. abreviaturas, unidades, formato de números etc.) en la Guía general para autores de Biota Colombiana.

5. Una vez incluida y verificada toda la información en el editor electrónico notifique al EC-SiB al correo electrónico sib+iac@ humboldt.org.co, indicando que ha finalizado la edición del manuscrito. Adicionalmente adjunte la plantilla de Excel con los datos estructurados (elimine todas las columnas que no utilizó). El EC-SiB realizará correcciones y recomendaciones finales acerca de la estructuración de los datos y dará las instrucciones finales para que usted proceda a someter el artículo.

\section{Someter el manuscrito}

Una vez haya terminado la edición de su manuscrito y recibido las instrucciones por parte del EC-SIB, envíe una carta al correo electrónico biotacol@humboldt.org.co para someter su artículo, siguiendo las instrucciones en la Guía general para autores de Biota Colombiana.

Recuerde adjuntar:

- Plantilla de Excel con la última versión de los datos revisada por el EC-SiB.

- Documento de Word con las figuras y tablas seguidas de una lista las mismas.

Cuando finalice el proceso, sus datos se harán públicos y de libre acceso en los portales de datos del SiB Colombia y GBIF. Esto permitirá que sus datos estén disponibles para una audiencia nacional e internacional, manteniendo siempre el crédito para los autores e instituciones asociadas. 
Anexo 1. Estructura base de un artículo de datos y su correspondencia con el editor electrónico basado en el GMP.

\begin{tabular}{|c|c|}
\hline SECCIÓN/SUBSECCIÓN & CORRESPONDENCIA CON LOS ELEMENTOS DEL EDITOR ELECTRÓNICO \\
\hline TÍTUlo & Derivado del elemento título. \\
\hline AutORES & Derivado de los elementos creador del recurso, proveedor de los metadatos y partes asociadas. \\
\hline AFILIACIONES & $\begin{array}{l}\text { Derivado de los elementos creador del recurso, proveedor de los metadatos y partes asociadas. } \\
\text { De estos elementos, la combinación de organización, dirección, código postal, ciudad, país y } \\
\text { correo electrónico, constituyen la afiliación. }\end{array}$ \\
\hline AUTOR DE CONTACTO & Derivado de los elementos creador del recurso y proveedor de los metadatos. \\
\hline CITACIÓN & Para uso de los editores. \\
\hline CitACión DELE RECURSO & Derivada del elemento referencia del recurso. \\
\hline RESUMEN & Derivado del elemento resumen. Máximo 200 palabras. \\
\hline Palabras Clave & Derivadas del elemento palabras clave. Máximo seis palabras. \\
\hline ABSTRACT & Derivado del elemento abstract. Máximo 200 palabras. \\
\hline KEY WORDS & Derivadas del elemento key words. Máximo seis palabras. \\
\hline INTRODUCCIÓN & $\begin{array}{l}\text { Derivado del elemento propósito (de las secciones Introducción y Antecedentes). Se sugiere un } \\
\text { breve texto para introducir las siguientes secciones. Por ejemplo, historia o contexto de la colección } \\
\text { biológica o proyecto en relación con los datos descritos, siempre y cuando no se repita información } \\
\text { en las subsecuentes secciones. }\end{array}$ \\
\hline Datos del proyecto & $\begin{array}{l}\text { Derivada de los elementos de la sección Datos del proyecto: título, nombre, apellido, rol, fuentes } \\
\text { de financiación, descripción del área de estudio y descripción del proyecto. }\end{array}$ \\
\hline Cobertura taxonómica & $\begin{array}{l}\text { Derivada de los elementos de la sección Cobertura taxonómica: descripción, nombre científico, } \\
\text { nombre común y categoría. }\end{array}$ \\
\hline Cobertura geográfica & $\begin{array}{l}\text { Derivada de los elementos de la sección Cobertura geográfica: descripción, latitud mínima, } \\
\text { latitud máxima, longitud mínima, longitud máxima. }\end{array}$ \\
\hline Cobertura temporal & Derivada de los elementos de la sección Cobertura temporal: tipo de cobertura temporal. \\
\hline Datos de la colección & $\begin{array}{l}\text { Derivada de los elementos de la sección Datos de la colección: nombre de la colección, } \\
\text { identificador de la colección, identificador de la colección parental, método de preservación } \\
\text { de los especímenes y unidades curatoriales. }\end{array}$ \\
\hline MATERIAL Y MÉTODOS & $\begin{array}{l}\text { Derivado de los elementos de la sección Métodos de muestreo: área de estudio, descripción del } \\
\text { muestreo, control de calidad, descripción de la metodología paso a paso. }\end{array}$ \\
\hline \multicolumn{2}{|r|}{${ }^{3}$} \\
\hline Descripción del conjunto de datos & $\begin{array}{l}\text { Derivado de los elementos de las secciones Discusión y Agradecimientos, contiene información } \\
\text { del formato de los datos y metadatos: nivel de jerarquía, fecha de publicación y derechos de } \\
\text { propiedad intelectual. }\end{array}$ \\
\hline Discusión & $\begin{array}{l}\text { Se deriva del elemento discusión. Un texto breve (máximo } 500 \text { palabras), que puede hacer } \\
\text { referencia a la importancia, relevancia, utilidad o uso que se le ha dado o dará a los datos en } \\
\text { publicaciones existentes o en posteriores proyectos. }\end{array}$ \\
\hline AGRADECIMIENTOS & Se deriva del elemento agradecimientos. \\
\hline BIBLIOGRAFÍA & Derivado del elemento bibliografía. \\
\hline
\end{tabular}


Anexo 2. Formatos para llenar el elemento referencia del recurso.

La referencia del recurso es aquella que acompañará los datos descritos por el artículo, públicos a través de las redes SiB Colombia y GBIF. Tenga en cuenta que esta referencia puede diferir de la del artículo. Para mayor información sobre este elemento contacte al EC-SiB. Aquí se sugieren dos formatos, sin embargo puede consultar otros formatos establecidos por $\mathrm{GBIF}^{4}$.

\section{TIPO DE RECURSO}

El conjunto de datos que el manuscrito describe es resultado de un proyecto de carácter institucional o colectivo con múltiples participantes.

\section{PLANTILLA}

$<$ Institución publicadora/ Grupo de investigación $>$ $<$ (Año) $>$, $<$ Título del recurso/Artículo $>$. $<$ Número total de registros $>$, <aportados por: $><$ parte asociada 1 (rol), parte asociada $2(\mathrm{rol})(\ldots)>$. $<$ En línea, $><$ url del recurso $>$. $<$ Publicado el DD/MM/ AAAA $>$.

\section{EJEMPLO}

Centro Nacional de Biodiversidad (2013). Vertebrados de la cuenca de la Orinoquia. 1500 registros, aportados por Pérez, S. (Investigador principal, proveedor de contenidos, proveedor de metadatos), M. Sánchez (Procesador), D. Valencia (Custodio, proveedor de metadatos), R. Rodríguez (Procesador), S. Sarmiento (Publicador), V. B. Martínez (Publicador, editor). En línea, http://ipt.sibcolombia.net/biota/resource.do?r=verte_orin, publicado el 01/09/2013.

Valencia, D., R. Rodríguez y V. B. Martínez (2013). Vertebrados de la cuenca del Orinoco. 1500 registros, en línea, http://ipt.sibcolombia.net/biota/ resource.do?r=verte orin. Publicado el 01/09/2001.
El conjunto de datos que el manuscrito describe es resultado de una iniciativa personal o de un grupo de investigación definido.
$<$ Parte asociada 1, parte asociada $2(\ldots)>$ $<$ (Año) $>$, $<$ Título del recurso/Artículo $>$, $<$ Número total de registros $>$, <en línea, $><$ url del recurso $>$. $<$ Publicado el DD/MM/AAAA $>$

\section{Guidelines for authors - Data Papers}

www.humboldt.org.co/es/bibliotecaypublicaciones/biota- biotacol@humboldt.org.co|

www.sibcolombia.net - sib+iac@humboldt.org.co

The purpose of this guide is to establish and explain the necessary steps to prepare a manuscript with the potential to become a publishable data paper in Biota Colombiana. This guide includes aspects related to the preparation of both data and the manuscript.

\section{What is a Data Paper?}

A data paper is a scholarly publication that has emerged as a mechanism to encourage the publication of biodiversity data as well as an approach to generate appropriate academic and professional recognition to all those involved in in the management of biodiversity information.

A data paper contains the basic sections of a traditional scientific paper. However, these are structured according to an international standard for metadata (information that gives context to the data) known as the GBIF Metadata Profile (GMP)5. The structuring of the manuscript based on this standard enables the community of authors publishing datasets globally, with presence in networks such as the Global Biodiversity Information Facility (GBIF) and other related networks, to publish data easily while getting proper recognition for their work and to encourage the authors of this type of data sets that have not yet published in these global information networks to have the necessary incentives to do so.

A data paper should describe in the best possible way the Whom, What, Where, When, Why and How of documenting and recording of data, without becoming the instrument to make a detailed analysis of the data, as happens in other academic publications. To deepen this publishing model, it is recommended to consult Chavan \& Penev (2011) ${ }^{6}$.

\footnotetext{
${ }^{4}$ GBIF (2012). Recommended practices for citation of the data published through the GBIF Network. Version 1.0 (Authored by Vishwas Chavan), Copenhagen: Global Biodiversity Information Facility. Pp.12, ISBN: 87-92020-36-4. Accessible at http://links.gbif.org/gbif_best_practice_data_citation_ en_v1

${ }^{5}$ GBIF (2011). GBIF Metadata Profile, Reference Guide, Feb 2011, (contributed by O Tuama, E., Braak, K., Copenhagen: Global Biodiversity Information Facility,19 pp. Accesible at http://links.gbif.org/gbif_metadata_profile_how-to_en_v1.

${ }^{6}$ Chavan, V. y L. Penev. 2011. The data paper: The mechanism to incentivize data publishing in biodiversity science. BMC Bioinformatics 12 (Suppl 15): S2.
} 
Guía para aut ores - Artículos de Datos / Guidelines for authors - Data Papers

\section{Which manuscripts are suitable for publication as data paper?}

Manuscripts that describe datasets containing original primary biological records (data of occurrences in a particular place and time); information associated with specimens of biological collections, thematic or regional inventories of species, genomic data and all data likely to be structured with the standard Darwin CoreDarwin Core $^{7}$ (DwC). This standard is used in the community of authors publishing biodiversity datasets to structure the data and thus to consolidate and integrate from different sources globally. It is not recommended to submit manuscripts describing secondary datasets, such as biological records compilations from secondary sources (e.g. literature or compilations of records already published in networks such as GBIF or IABIN).

\section{Dataset preparation}

As mentioned above data submitted in this process should be structured based on DwC standard. For ease of structuring, the Biodiversity Information System of Colombia (SiB Colombia), created two templates in Excel; one for occurrences and other for species checklist. Carefully read and follow the template instructions for structuring and publishing data. For any questions about the structure process of data please contact the Coordinator Team of SiB Colombia (EC-SiB) at sib+iac@humboldt.org.co

\section{Manuscript preparation}

To assist the creation and structuring of the manuscript in the GMP standard, an electronic writing tool is available (http://ipt. sibcolombia.net/biota) to guide the author in the process and ultimately generate a first version of the manuscript. The use of GMP manual as an information guide to include in each section of the manuscript, as well as the annex 1 is recommended.

Steps required for the manuscript preparation:

1 Request access to the electronic writing tool at sib+iac@ humboldt.org.co. The EC-SiB will assign a username and password.

2. Login to the electronic writing tool, then go to the tab Manage Resources and create a new resource by assigning a short name for your manuscript and clicking on the Create button. Use the format: "InstitutionAcronym_Year_DatasetFeature", e.g. NMNH 2010 rainforestbirds.

3. In the overview of the writing tool click on edit in Metadata section (please, do not use any other section), once there you will find different sections (right panel) that will guide you creating your manuscript. Save the changes at the end of each section, otherwise you will lose the information. Remember to use the GMP manual. Here are some recommendations for editing the metadata, sections are indicated in CAPS and the elements of these sections in bold.
- In ASSOCIATED PARTIES include only those who are not listed in BASIC INFORMATION.

- PROJECT DATA and COLLECTION DATA are optional depending on the data type. When using these sections extend or complement information already provided, i.e. do not repeat the same information describing the description (GEOGRAPHIC COVERAGE) in the study area description (PROJECT DATA).

- Likewise, in SAMPLING METHODS, you must expand or complete the information, not repeat it. The information in study extent should give a specific context of the sampling methodology.

- It is essential to document the quality control in SAMPLING METHODS. Here you should describe what tools or protocols were used to ensure the quality and consistency of data structured with DwC standard.

- To create the resource citation in the CITATIONS section, follow one of the two formats proposed (Annex 2). Do not fill out the citation identifier, this will be provided later by the EC-SiB.

- To include the manuscript bibliography in citations, enter each of the citations individually, adding a new citation each time by clicking in the bottom left.

4. Check that the format of the information provided meets the guidelines of the journal (e.g. abbreviations, units, number formatting, etc.) in the Biota Colombiana Guidelines for Authors.

5. Once included and verified all information in the writing tool, notify to EC-SiB at sib+iac@humboldt.org.co, indicating that you have finished editing the manuscript. Additionally attach the Excel template with structured data (remove all columns that were not used). The EC-SiB will perform corrections and final recommendations about the structure of the data and give you the final instructions to submit the paper.

\section{Submit the manuscript}

Once you have finished editing your manuscript and getting the instructions from EC-SIB, send a letter submitting your article to email biotacol@humboldt.org.co, following the instructions of Biota Colombiana Guidelines for Authors.

Remember to attach:

- Excel template with the latest version of the data reviewed by the EC-SiB.

- Word document with figures and tables followed by a list of them.

At the end of the process, your information will be public and freely accessible in the data portal of SiB Colombia and GBIF. This will allow your data to be available for national and international audience, while maintaining credit to the authors and partner institutions.

\footnotetext{
${ }^{7}$ Biodiversity Information Standards - TDWG. Accesible at http://rs.tdwg.org/dwc/terms/
} 
Annex 1. Basic structure of a data paper and its mapping to the writing tool elements based on GM.

\begin{tabular}{|c|c|}
\hline $\begin{array}{l}\text { SECTION/SUB-SECTION } \\
\text { HEADING }\end{array}$ & MAPPING WITH WRITING TOOL ELEMENTS \\
\hline TitLE & Derived from the title element. \\
\hline Authors & Derived from the resource creator, metadata provider, and associated parties elements. \\
\hline AFFILIATIONS & $\begin{array}{l}\text { Derived from the resource creator, metadata provider and associated parties elements. From } \\
\text { these elements combinations of organization, address, postal code, city, country and email } \\
\text { constitute the affiliation. }\end{array}$ \\
\hline CORRESPONDING AUTHOR & Derived from the resource contact, metadata provider elements. \\
\hline CITATION & For editors use. \\
\hline RESOURCE CITATION & Derived from the resource citation element. \\
\hline RESUMEN & Derived from the resumen element. 200 words max. \\
\hline Palabras Clave & Derived from the palabras clave element. 6 words max. \\
\hline ABSTRACT & Derived from the abstract element. 200 words max. \\
\hline KEY WORDS & Derived from the key words element. 6 words max. \\
\hline INTRODUCTION & $\begin{array}{l}\text { Derived from the purpose (Introduction and Background section). A short text to introduce the } \\
\text { following sections is suggested. For example, history or context of the biological collection or } \\
\text { project related with the data described, only if that information is not present in subsequent sections. }\end{array}$ \\
\hline Project data & $\begin{array}{l}\text { Derived from elements title, personnel first name, personnel last name, role, funding, study } \\
\text { area description, and design description. }\end{array}$ \\
\hline Taxonomic Coverage & $\begin{array}{l}\text { Derived from the taxonomic coverage elements: description, scientific name, common name } \\
\text { and rank. }\end{array}$ \\
\hline Geographic Coverage & Derived from the geographic coverage elements: description, west, east, south, north. \\
\hline Temporal Coverage & Derived from the temporal coverage elements: temporal coverage type. \\
\hline Collection data & $\begin{array}{l}\text { Derived from the collection data elements: collection name, collection identifier, parent } \\
\text { collection identifier, specimen preservation method and curatorial units. }\end{array}$ \\
\hline MATERIALS AND METHODS & $\begin{array}{l}\text { Derived from the sampling methods elements: study extent, sampling description, quality } \\
\text { control and step description. }\end{array}$ \\
\hline \multicolumn{2}{|l|}{ RESULTADOS } \\
\hline Descripción del conjunto de datos & $\begin{array}{l}\text { Derived from the discussion and acknowledgments, contains information about the format of the } \\
\text { data and metadata: hierarchy level, date published and ip rights. }\end{array}$ \\
\hline DisCUSSION & $\begin{array}{l}\text { Derived from the discussion element. A short text ( } \max 500 \text { words), which can refer to the } \\
\text { importance, relevance, usefulness or use that has been given or will give the data in the published } \\
\text { literature or in subsequent projects. }\end{array}$ \\
\hline ACKNOWLEDGMENTS & Derived from the acknowledgments element. \\
\hline BIBLIOGRAPHY & Derived from the citations element. \\
\hline
\end{tabular}


Annex 2. Citation style quick guide for "resource reference" section.

The Resource Reference is the one that refer to the dataset described by the paper, publicly available through SiB Colombia and GBIF networks. Note that this reference may differ from the one of the paper. For more information about this element contact EC-SiB.

Here two formats are suggested; however you can consult other formats established by GBIF .

\section{TYPE OF RESOURCE}

The paper is the result of a collective or institutional project with multiple participants.

The paper is the result of a personal initiative or a defined research group.

\section{TEMPLATE}

$<$ Institution/Research Group $>$. < Year $>,<$ Title of the Resource/Paper $>$. $<$ Number of total records $>$, <provided by :> <associated party 1 (role), associated party 2 (role), (...) > $<$ Online, $>$ $<$ resource URL $>$, $<$ published on $>$. $<$ Published on DD/MM/AAAA $>$.

$<$ associated party 1 , associated party $2,(\ldots)>$. $<$ Year $>$, $<$ Title of the Resource/Paper $>$, $<$ Number of total records $>,<$ Online, $><$ resource URL $>$. $<$ Published on DD/MM/AAAA $>$.

\section{EXAMPLE}

National Biodiversity (2013). Vertebrates in Orinoco, 1500 records, provided by: Perez, S. (Principal investigator, content provider), M. Sanchez (Processor), D. Valencia (Custodian Steward, metadata provider), R. Rodriguez (Processor), S. Sarmiento (Publisher), VB Martinez (Publisher, Editor). Online, http://ipt.sibcolombia.net/ biota/resource.do? $\mathrm{r}=$ verte orin, published on 01/09/2013.

Valencia, D., R. Rodríguez and V. B. Martínez. (2013). Vertebrate Orinoco Basin, 1500 records, Online, http://ipt.sibcolombia.net/biota/resource. do? $\mathrm{r}=$ verte_orin, published on 01/09/2001

${ }^{8}$ GBIF (2012). Recommended practices for citation of the data published through the GBIF Network. Version 1.0 (Authored by Vishwas Chavan), Copenhagen: Global Biodiversity Information Facility. Pp.12, ISBN: 87-92020-36-4. Accessible at http://links.gbif.org/gbif_best practice_data_citation en $\mathrm{v} 1$ 


\section{TABLA DE CONTENIDO / TABLE OF CONTENTS}

Aguas subterráneas, humedales y servicios ecosistémicos en Colombia. Groundwater, wetlands and ecosystem services in Colombia. Teresita Betancur-Vargas, Daniel A. García-Giraldo, Angélica J. Vélez-Duque, Angélica M. Gómez, Carlos Flórez-Ayala, Jorge Patiño y Juan Á. Ortiz-Tamayo

Efecto del $\mathrm{CaCl}_{2}$ sobre el contenido de proteínas, prolina, acidez titulable, clorofila y contenido relativo de agua de $A$ loe vera expuesta a salinidad por $\mathrm{NaCl}$. $\mathrm{CaCl}_{2}$ effect on protein, proline, titratable acidity, chlorophyll and relative water content from Aloe vera exposed to salinity by NaCl. Selwin Pérez-Nasser .... Efecto del $\mathrm{Ca}^{2+}$ sobre algunas variables de crecimiento de Aloe vera cultivada con $\mathrm{NaCl}$. Effect of $\mathrm{Ca}^{2+}$ on some growth variables from Aloe vera grown on $\mathrm{NaCl}$. Selwin Pérez-Nasser

Charophyta, Chlorophyta y Cryptophyta del embalse Riogrande II (Antioquia), Colombia. Charophyta, Chlorophyta and Cryptophyta in Riogrande II reservoir (Antioquia), Colombia. Mónica T. López Muñoz, Carlos E. De Mattos-Bicudo, Ricardo O. Echenique, John J. Ramírez-Restrepo y Jaime A. Palacio ................ Diferencias del contenido nutricional de hojas jóvenes y maduras de dos especies de puya (Puya santosii Cuatrec., Puya goudotiana Mez; Bromeliaceae), en la región del Guavio, Cundinamarca, Colombia. Differences in the nutritional content of mature and young Puya leaves (Puya santosii Cuatrec., Puya goudotiana Mez; Bromeliaceae) in the Guavio region, Cundinamarca, Colombia. Luis J. Romero-Puentes, Brayan L. Torres-Clavijo y Angela ParradoRosselli

Características físicas y germinativas de semillas de la orquídea Prosthechea sp. de la zona andina, Fusagasugá, Colombia. Physical and germinative characteristics of Prosthechea sp. (Orchidaceae) native to Fusagasugá - Colombia. Laguandio del C. Banda-Sánchez, Yeison H. Pinzón-Ariza y Luis E. Vanegas-Martínez

Especies vegetales colonizadoras de áreas perturbadas por la minería en bosques pluviales del Chocó, Colombia. Colonizer plant species of sites disturbed by mining in the Chocoan rain forests, Colombia. Hamleth Valois-Cuesta y Carolina Martinez-Ruiz

Catálogo de la flora vascular de los Parques Nacionales de Colombia: Santuario de Flora y Fauna de Iguaque y su zona de amortiguamiento. Catalog of the vascular flora of the National Parks of Colombia: Iguaque Fauna and Flora Sanctuary and buffer zone. Humberto Mendoza-Cifuentes .....

Cambios estructurales del mesozooplancton en relación a las condiciones hidrográficas en el golfo de Cariaco, Venezuela. Structural changes of mesozooplankton in relation to hydrographic conditions in the Gulf of Cariaco, Venezuela. Brightdoom Márquez-Rojas, Evelyn Zoppi de Roa, Luis Troccoli y Edy Montiel

Chinches patinadoras marinas (Hemiptera: Heteroptera: Gerromorpha): diversidad de los hábitats oceánicos del Neotrópico. Marine water striders (Hemiptera: Heteroptera: Gerromorpha): diversity of ocean habitats in the Neotropics. Fredy Molano-Rendón e Irina Morales

Descripción de una nueva especie de mariposa del género Wahydra Steinhauser (Lepidoptera: Hesperiidae: Hesperiinae: Anthoptini) para Colombia. Description of a new species of butterfly of the genus Wahydra Steinhauser (Lepidoptera: Hesperiidae: Hesperiinae: Anthoptini) from Colombia. Efrain R. Henao-Bañol, Fabián G. Gaviria y Julián A. Salazar-Escobar

Pseudoescorpiones (Arachnida: Pseudoscorpiones) del nororiente andino de Colombia. Pseudoscorpions (Arachnida: Pseudoscorpiones) in the norteastern Andean region of Colombia. Catalina Romero-Ortiz

Primer registro de cuatro especies de camarones de agua dulce (Palaemonidae) para Colombia. First records of four species of freshwater shrimp (Palaemonidae) from Colombia. Ada Acevedo y Carlos A. Lasso

Lista anotada de los tipos de peces en la colección del Laboratorio de Ictiología, Universidad del Quindío, Armenia, Colombia (IUQ). Annotated list of types of fishes in the collection of the Laboratory of Ichthyology, University of Quindío, Armenia, Colombia (IUQ). César Román-Valencia, Donald C. Taphorn, Carlos. A. García-Alzate, Sebastián Vásquez-P. y Raquel I. Ruiz-C........

Pterygoplichthys undecimalis (Siluriformes: Loricariidae): una especie trasplantada en la cuenca del río Patía, vertiente Pacífico, Colombia. Pterygoplichthys undecimalis (Siluriformes: Loricariidae): a species transplanted to the Basin of the Patía River, Colombia. Alberto Moncayo-Fernández, Ofelia Mejía-Egas y Héctor E. Ramirez-Chaves

Lista anotada de la herpetofauna del departamento del Quindío, Colombia. Checklist of the herpetofauna of the department of Quindío, Colombia. Cristian Román-Palacios, Sara Fernández-Garzón, Alejandro Valencia-Zuleta, Andrés F. Jaramillo-Martínez y Ronald A. Viáfara-Vega

Batracofauna de los bosques de niebla y estribaciones del piedemonte en el municipio de Yopal (Casanare), Orinoquia colombiana. Frogs and toads of cloud forests and foothills in the Yopal municipality (Casanare), Colombia. Andrés R. Acosta-Galvis

Jagüeyes y su papel potencial en la conservación de tortugas continentales en el golfo de Morrosquillo, Sucre, Caribe colombiano. Cattle ponds and their potential role in conservation of freshwater turtles in the Gulf of Morrosquillo, Sucre, Colombia. Jaime De La Ossa-V., Merly Ardila-Marulanda, Alejandro De La Ossa-Lacayo

Aspectos poblacionales de primates diurnos simpátricos que habitan parches de bosque seco tropical en los Montes de María, Sucre, Colombia. Populational aspects of diurnal sympatric primates inhabiting patches of tropical dry forest in the Montes de María, Sucre, Colombia. Jaime De La Ossa-V. $y$ Silvia Galván-Guevara

Diversidad de pequeños mamíferos no voladores (Didelphimorphia, Paucituberculata y Eulipotyphla) en Áreas de Protección Estricta de Venezuela. Diversity of non-volant small mammals (Didelphimorphia, Paucituberculata and Eulipotyphla) in the Strictly Protected Areas in Venezuela. Franger J. García, Mariana I. Delgado-Jaramillo y Marjorie Machado

La integridad biológica como herramienta de valoración cuantitativa del estado de conservación del bosque seco en Colombia. Biological integrity as a tool for quantitative assessment of the conservation status of dry forest in Colombia. Wilmar Bolivar-García, Alan Giraldo y Ángela M. González-Colorado

Nota

Ampliación de la distribución geográfica de Microgenys minuta Eigenmann 1913 (Characiformes, Characidae) en la cuenca del río Magdalena, Colombia. Expansion of distribution of Microgenys minuta Eigenmann 1913 (Characiformes, Characidae) in the Magdalena River basin, Colombia. Lina M. Mesa-S. $y$ Juan G. Albornoz

\section{Artículo de datos}

Colección Ictiológica de la Universidad Industrial de Santander, Colombia. Ichthyology Collection of the Industrial University of Santander, Colombia. Mauricio Torres, Egna Mantilla-Barbosa, Federico Rangel-Serpa.....

Guía para autores. Guidelines for authors 